\title{
Design of fiber-integrated tunable thermo-optic C-band filter based on coated silicon slab
}

\author{
H. Pinhas ${ }^{1 *} \mathbb{D}$, D. Malka², Y. Danan', M. Sinvani ${ }^{1}$ and Z. Zalevsky
}

\begin{abstract}
Background: Optical filters are required to have narrow band-pass filtering in the spectral C-band for applications such as signal tracking, sub-band filtering or noise suppression. These requirements lead to a variety of filters that offer

thermo-optic effect for optical switching, however, some of which without proper thermal and optical efficiency.

Methods: In this paper we propose a tunable thermo-optic filtering device based on a coated silicon slab resonator with an increased Q-factor to allow for an efficient thermo-optical switching in the C-band. The device can be designed either for long range wavelength tuning or for short range with increased wavelength resolution.

Results: Theoretical examination of the thermal parameters affecting the filtering process is shown together with experimental results. Proper channel isolation with an extinction ratio of $20 \mathrm{~dB}$ is achieved with a spectral bandpass width of $0.07 \mathrm{~nm}$.

Conclusions: This Si slab based filter characteristics make it suitable for wavelength switching systems such as dense wavelength division multiplexing. In addition, the filter is fiber integrated in order for it to be more compatible with other optics communication devices.
\end{abstract}

Keywords: Optoelectronics, Switching, Resonators, Infrared systems

\section{Background}

Many optical devices have been proposed as an Infinite Impulse Response (IIR) filter [1-3]. Some of these devices implementation has been suggested as ring resonators and have been implemented with Silicon-OnInsulator (SOI) [4-6]. The IIR resonator implementation as a filter is only one among many implemented applications [7-11]. Such applications are often used for spectral ranges around $1530-1565 \mathrm{~nm}$ which is defined as the Cband. These filtering devices offer a narrow band-pass, in the full C-band region, which is required for applications such as signal tracking, sub-band filtering and noise suppression [3, 12-15]. In addition, because of the implementation in the spectral C-band region, these filters can be integrated in optical communication networks.

\footnotetext{
*Correspondence: hadarpinhas@gmail.com

${ }^{1}$ Faculty of Engineering and the Nano-Technology Center, Bar-llan University, 5290002 Ramat Gan, Israel

Full list of author information is available at the end of the article
}

Recently, a finite impulse response (FIR) filter has been implemented with a built-in IIR resonator in the form of racetrack resonator [16]. Racetrack resonators, among other IIR resonators, are based upon constructive interference effect, in which the optical path length of the resonator is exactly an integer multiplication of half wavelength of the incident laser beam wavelength. Whereas the FIR resonators are discreet-time filters with impulse response that contains a finite number of coefficients and their design is often based upon Mach-Zehnder interferometer and coupler components $[17,18]$.

The IIR resonator plays an important role in the filters application field; the advantage of IIR filter resonators over the FIR filters stems from the sensitivity of the filter which is greater in IIR resonator and expressed in its high finesse, or Q factor [19]. The sensitivity is defined by how much shift in wavelength does it takes to decrease the transmission from its maximum. The higher the finesse, the less wavelength shift is needed to decrease the transmission to 
the same lower transmission. This, together with a simplicity of a device made out of only one resonator rather than other options, such as several concatenated chain of MachZehnder interferometers give its advantage.

One important restriction in designing an IIR filter is its realization with $\mathrm{Si}$ in order to allow its integration with microelectronic circuitry. In addition, Si thermooptic coefficient $\left(\mathrm{TOC}=1.86 \times 10^{-4} 1 / \mathrm{K}\right)$ is larger by an order of magnitude than that of silica $\left(10^{-5} 1 / \mathrm{K}\right)$, used in optical fibers; both with specific heat capacity of about $700 \mathrm{~J} /\left(\mathrm{kg}^{*} \mathrm{~K}\right)$. In a simulation of the thermo-optic effect (TOE) in silica, thermal change of $500 \mathrm{~K}$ is needed in order to change its refraction index by $5 \times 10^{-3}$ refractive index units [20], instead of less than $50 \mathrm{~K}$ with silicon. The vast change in temperature can induce involuntary changes in the material parameters such as the change in the free spectral range (FSR) [21]. Hence, with a larger TOC and about the same specific heat capacity, the amount of heat or power required to induce a change in the system with $\mathrm{Si}$ instead of silica is lower.

One application of the IIR resonator is an all-optical comb switch for multi-wavelength message routing in photonic networks, which is implemented as a ring resonator [22]. Although silicon ring resonators and in some cases Fabry-Perot (FP) resonators [23], can have high finesse they either require complex structures and metal depositions in highly defined manner or cannot be fabricated in in-fiber applications such as in-fiber modulators since they have to be implemented as part of an SOI chip [24-28]. Even though ring resonators can be made in e.g. InP without any silicon/silica and does not require SOI, they will lack the advantages of silicon such as low price, industry oriented and easier integration in silicon based integrated circuits. Indeed, the coated silicon slab can be regarded as not less complicated, however, since it does not require lithography, etching, annealing or any other process, but only deposition of layers it is said to be less complicated. FB resonator can have high finesse with coating or in other ways, such as shown in [8], in which they propose by recording Bragg mirrors on both sides of the silicon inside a fiber, as discussed in details in the discussion section with a $1 \mathrm{~dB}$ insertion loss.

Rather than using a ring resonator, one can implement the IIR resonator as a simple FP resonator. One way to implement it is with a Si slab which has a simpler structure and is SOI independent. The main problem with a Si slab IIR resonator as a filter is that its finesse is low. The finesse, determined by the reflectivity of the slab planes, of a bare silicon slab is about 2.5 with reflectivity of 0.3 , which in turn is determined by the high refractive index of 3.5 compared to air, with refractive index of 1 . Thus, by increasing the reflectivity of the slab, for instance with coatings, the finesse can increase. Increased finesse is manifested in increased sensitivity to any changes in refractive index as described in details below. With increased sensitivity light passing through the silicon slab, tuned to slab resonance, will be prone to decreased intensity with smaller refractive index changes. In addition, the band width of a resonator with increased finesse is narrower than in a low finesse one, that is, the destructive and constructive interferences will have higher influence on the light intensity of a higher finesse. Therefore, a bare Si slab has a modulation depth of up to $70 \%$ or $5 \mathrm{~dB}$, which will be lower than an improved finesse one. In addition, its full width half maximum (FWHM) is about half its FSR which yields a very poor filter compared to high finesse slab.

In this paper, we present a thermo-optic tunable IIR filter based on a coated $\mathrm{Si}$ slab for the C-band region. The coated Si slab has significantly improved characteristics over a bare Si such as high modulation depth and low power consumption that are obtained due to the coatings. It has a narrow and smooth transmission window which provides good channel isolation. The bandwidth is shown to be $0.07 \mathrm{~nm}$ or almost $9 \mathrm{GHz}$. The coated Si slab improves the transmission performance over a bare $\mathrm{Si}$, as was discussed and detailed in our previous work [29]. This paper is a follow-up work in which the slab improved performance is utilized in an in-fiber application i.e. integrated in-fiber optics communication system filter. The simplicity of the proposed device gives it an advantage over other IIR filters such as ring resonators by enabling it to be integrated between two fibers, avoiding the SOI structures and coupling of tapered waveguides. The characteristics of the proposed device, shown here after, make it suitable for wavelength switching systems such as dense wavelength division multiplexing (DWDM). The novelty of this paper is also in the fact that this Si slab filter can be a fiber integrated device to be interfaced with other optics communication fibers and fiber based devices.

\section{Methods}

Modulation of the filter is achieved by changing the real part of the refractive index of the $\mathrm{Si}$ [30]. This can be accomplished by various techniques: Electro-optical Kerreffect, optical Kerr-effect (such as self-modulation), stress, temperature (TOE), acousto-optical modulation and plasma dispersion effect PDE. We choose to focus on two techniques, PDE and TOE. The first is through a change in the free charge carriers (FCCs) concentration with their injection or through the generation of FCCs. The second can be obtained with temperature variation which modifies the real refractive index, which is the one mainly addressed in this paper. The change in the forbidden gap with temperature governs the changes in 
the refractive index via Kramers-Kronig relations, thus, affecting the absorption together with the phase (real refractive index). However, the advantage of the TOE over the PDE is that the absorption is lower and even negligible, as can be seen in the results section and in $[18,31]$.

The TOE theorem describes a direct impact on the real refractive index by the TOC in $\mathrm{Si}$ [32]:

$$
\frac{\mathrm{dn}}{\mathrm{dT}}=8.61 \times 10^{-5}+3.63 \times 10^{-7} \times T-2.07 \times 10^{-10} \times T^{2}
$$

where $\mathrm{n}$ is the real refractive index and $\mathrm{T}$ is the absolute temperature. That is, a rise in temperature of ten degrees around room temperature (RT) induces an increase of 0.001 in refractive index. The proposed device temperature range requirements are $350 \mathrm{~K}$ for the entire $\mathrm{C}$-band, however, around only $10 \mathrm{~K}$ will be sufficient for about $1 \mathrm{~nm}$ wavelength shift such as in the feedback loops application [33]. Since the most commonly used grid spacing for DWDM systems, from those defined by the International Telecommunication Unit Telecommunication Standardization Sector (ITU-T), is around $0.8 \mathrm{~nm}$, it could also be used with our method.

Since a Si slab has a high refractive index value $\sim 3.5$, the characteristics of a Si FP resonator has a finesse value of 2.5. The normalized transmission of a lossless FP resonator can be described by the Airy function:

$$
\frac{I_{\text {out }}}{I_{\text {in }}}=\frac{\left(1-R_{1}\right)\left(1-R_{2}\right)}{\left(1-\sqrt{R_{1}} \sqrt{R_{2}}\right)^{2}+4 \sqrt{R_{1}} \sqrt{R_{2}} \sin ^{2}\left(\frac{\delta}{2}\right)}
$$

where $R_{1}$ and $R_{2}$ are the reflectivity of the FP planes, $\delta$ $(=2 \pi \mathrm{nl} / \lambda)$ is the round trip phase, $\lambda$ is the laser beam wavelength in vacuum and $l$ is the silicon slab thickness. The transmission spectrum of the resonator is controlled by the phase shift $(\delta / 2)$ which is determined by the refractive index $\mathrm{n}=\mathrm{n}_{0}+\Delta \mathrm{n}$, where $\mathrm{n}_{0}$ is the refractive index at RT and $\Delta \mathrm{n}$ is the change in refractive index due to changes in temperature. The TOE influence on the transmission can be greatly increased with the increase in the finesse. Higher finesse is manifested by a narrower FWHM. A nominal temperature change induces a specific shift in wavelength which is independent of the finesse. Thus, with higher finesse the wavelength shift of the resonator does not change but the transmission decreases because of the new transmission spectrum. For instance, in Fig. 3a, the transmission is at its maximum at $1546 \mathrm{~nm}$ (blue solid line) and a temperature change induces a $0.116 \mathrm{~nm}$ shift so the transmission at this wavelength decreases (red dashed line). However, if the finesse was lower, that is, broader FWHM, the transmission would not have been decreased that much. That is, the sensitivity to temperature would have been lower and vice versa with higher finesse. In this manner, higher finesse is expressed in higher sensitivity to temperature, that is, higher sensitivity to signal tracking and filtering. Therefore, the coatings were added on both sides of the Si slab (see Fig. 2) to increase the finesse in the proposed device.

The temperature required for full FSR scan, or peak to peak wavelength shift, $\Delta T_{\pi}$, is dependent on the thickness of the FP, l, the TOC and the thermal expansion as described in the following eq. [31]:

$$
\Delta T_{\pi}=\frac{\lambda}{2 l\left(\frac{\partial n}{\partial T}+n k\right)}
$$

where $\mathrm{k}(=2.6 \mathrm{X} 10-61 / \mathrm{K})$ is the thermal expansion coefficient. The meaning of $\Delta \mathrm{T}_{\pi}$ can be better explained in the following sentences. A change in temperature induces a change in the real refractive index of the silicon. A change in the latter changes the transmission spectrum; with increase in temperature the transmission spectrum shifts to higher wavelengths (red shift), so light with higher wavelength is transmitted. In other words, a change in the transmission spectrum affects the transmitted intensity of the probe laser. That is, if the probe laser intensity is at its maximum at a nominal temperature and because of a continuous temperature change its intensity decreases and then returns to its maximum again the temperature change is said to be $\Delta \mathrm{T}_{\pi}$. If the probe laser intensity is at its maximum at a nominal temperature and because of a continuous temperature change its intensity decreases and does not return to its maximum the temperature change is not $\Delta \mathrm{T}_{\pi}$ but only a fraction of it. An example of a change in temperature that is only a fraction of $\Delta \mathrm{T}_{\pi}$ can be seen in Fig. 4. It this figure the temperature change is only $100 \mathrm{~K}$ where the $\Delta \mathrm{T}_{\pi}=350 \mathrm{~K}$, that is, $350 \mathrm{~K}$ is the temperature needed to for the probe laser to return to maximal transmission intensity.

The coatings were made of 18 dielectric layers of $\mathrm{Ta}_{2} \mathrm{O}_{5}$ and $\mathrm{SiO}_{2}$ with total coatings thickness of $\mathrm{d}=$ $4 \mu \mathrm{m}$ on each side of the slabs which were double sided optically polished, as seen in Fig. 1b. The coatings expected reflectivity in the IR is about $95 \%$ (finesse of 60), however, the highest finesse observed is 30 probably because of the silicon slab low surface quality. These coatings were chosen to reflect IR light (around $1550 \mathrm{~nm}$ ) for improved finesse and to transmit $532 \mathrm{~nm}$ and $808 \mathrm{~nm}$ (laser wavelengths at our disposal) for obtaining better pump efficiency. However, no optical pump was used in the research presented in this paper.

The experimental setup is composed of an IR laser beam, the probe, transmitted through the coated Si slab with beam direction normal to the slab face for maximum transmission intensity, as seen in Fig. 1a. The probe is a "HP" $5 \mathrm{~mW}$, wavelength tunable, $\mathrm{CW}$ laser beam around $1550 \mathrm{~nm}$ (model "HP 8168F") with $2 \mathrm{~mm}$ 

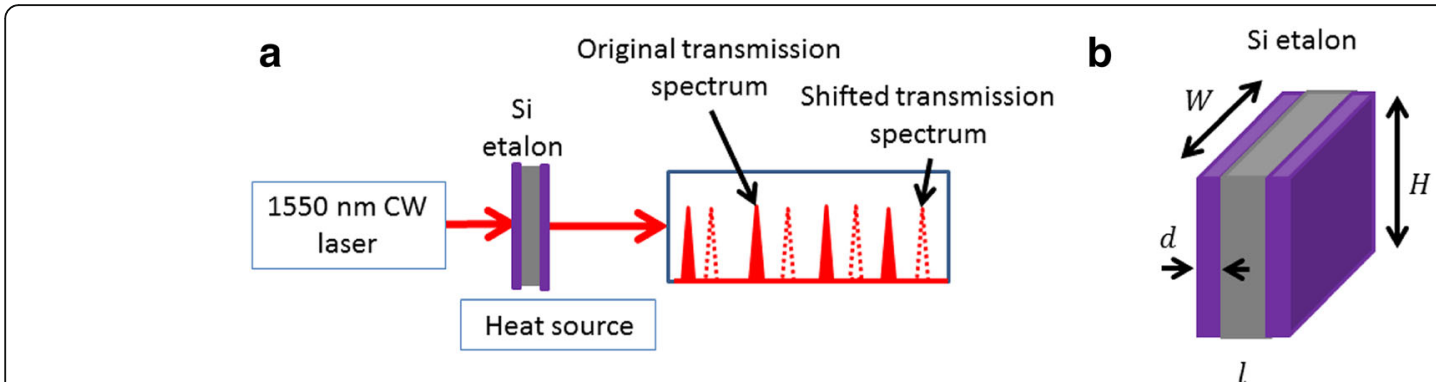

Fig. 1 a Experimental setup: transmission of IR laser beam trough the coated Si slab. b Si etalon dimensions: Width $=$ Height $=20 \mathrm{~mm}$, silicon thickness $\mathrm{I}=470 \mu \mathrm{m}$ and coating layers $\mathrm{d}=4 \mu \mathrm{m}$

diameter. The probe transmission is detected by "Thorlabs" InGaAs detector (model "PDA400") and the transmission spectrum is taken by an oscilloscope. The specific pass bands sensitivity is determined by the FWHM size which is dependent on the finesse acquired. The wavelength of the transmission bands are tuned by temperature variation, as mentioned before.

The transmission spectrum is shifted with the change in temperature which is controlled by a thermo-electric cooler (TEC) derived by "Wavelength Electronics" temperature controller (model "LFI-3751") with temperature control of $0.01 \mathrm{~K}$. The tuning time is few seconds but can be decreased to few micro-seconds with resistive heating and even shorter with optical heating [29, 34]. Even though optical heating can induce absorption, the real refractive index effect on a resonator transmission is usually larger than the increase in absorption [35]. In addition, the illumination of pump does not have to be in the area of transmitted signal, or probe, but rather in any other area from which heat can be conducted across the silicon sample (silicon is a good heat conductor), farther than the FCCs diffusion length, so absorption will be avoided. Figure 2 illustrates the filter tuning with the TEC. The total loss in this system is as low as $3 \mathrm{~dB}$, due to the sample coatings.

\section{Results}

\section{Simulations}

A Matlab simulation of the normalized transmission spectrum through a $\mathrm{Si}$ etalon $(470 \mu \mathrm{m}$ thick) as a function of wavelength at different temperatures can be seen in Fig. 3a. It is based on eqs. (1)-(3), being the TOE theorem which predicts the change in the transmission spectrum due to temperature variation. The simulation results show the dependence of wavelength shift on temperature at RT
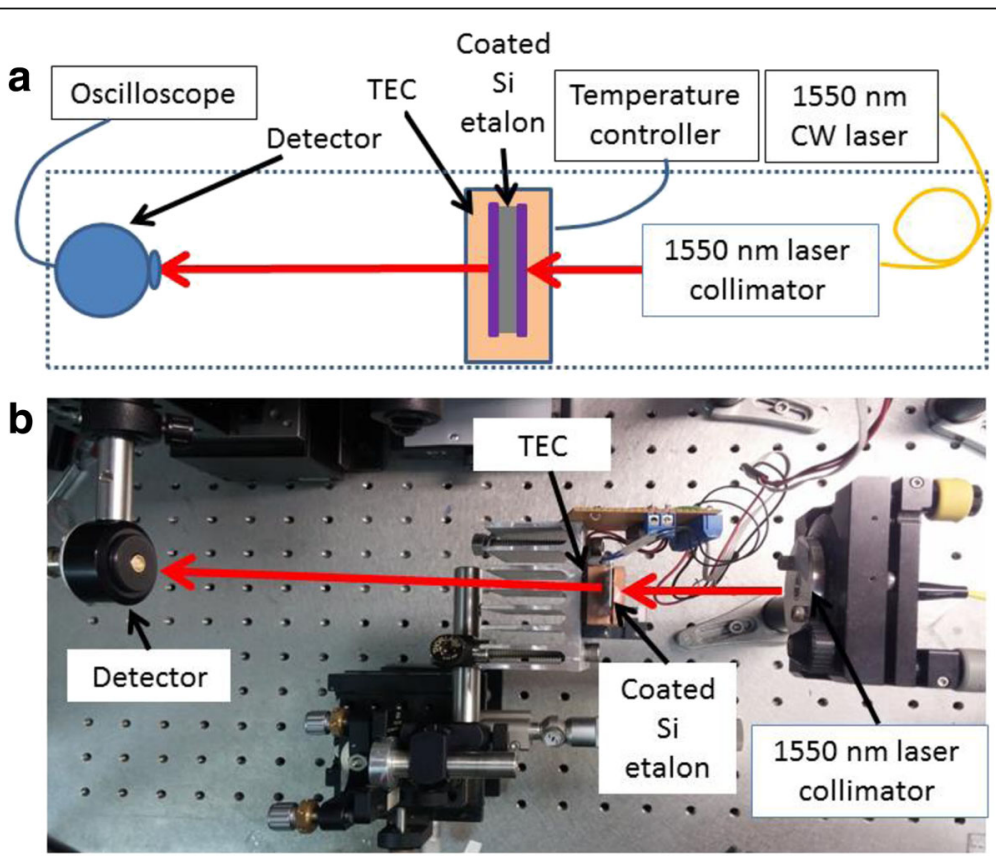

Fig. 2 The experimental setup: a Scheme b Picture 

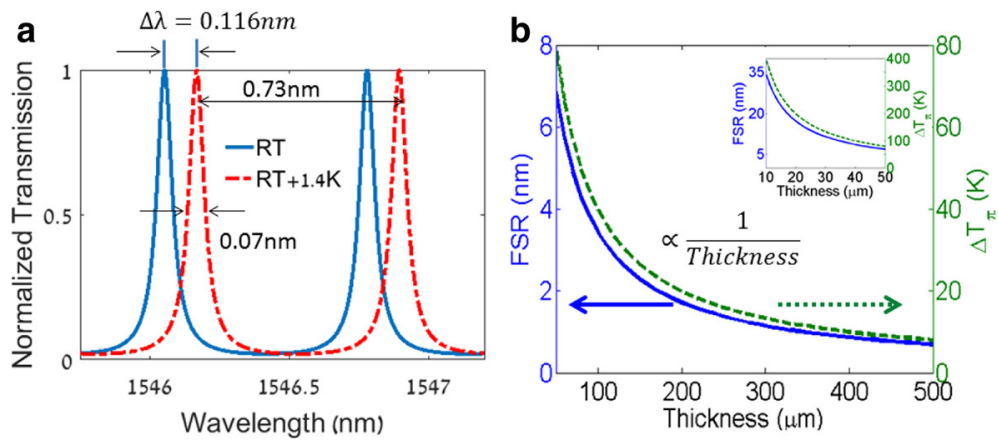

Fig. 3 Simulations: a Transmission of a coated Si slab (470 $\mu$ m thick) vs. wavelength at two temperatures. $\mathbf{b}$ FSR and $\Delta T_{\pi}$ vs slab thickness. Inset: Thickness variation yielding FSR of $35 \mathrm{~nm}$

(solid blue line) and above RT (dashed red line). The temperature variation chosen in the simulation is $1.4 \mathrm{~K}$ above RT which yields a wavelength shift of $0.116 \mathrm{~nm}$ which is a little more than the FWHM. With these results the dependence of wavelength shift on temperature is calculated to be $0.083 \mathrm{~nm} / \mathrm{K}$ and its shows that the full FSR can be covered by about $9 \mathrm{~K}$.

In Fig. 3b, simulation of the FSR and $\Delta \mathrm{T}_{\pi}$ as a function of thickness can be seen. Noticeably, the FSR (and consequently the FWHM) are inversely proportional to the thickness of the slab and so does the $\Delta \mathrm{T}_{\pi}$. Thus, for higher spectral resolution in short regions of wavelengths thinner slabs are needed. The opposite goes for scanning broad regions of wavelengths but with lower spectral resolution which requires thicker slabs.

In the inset of Fig. 3b, FSR of up to the full C-band region of $35 \mathrm{~nm}$ as a function of the thickness can be seen. It shows that a $10 \mu \mathrm{m}$ slab thick is required for FSR of $35 \mathrm{~nm}$ with $\Delta \mathrm{T}_{\pi}$ of about $350 \mathrm{~K}$. Therefore, a simulation of IR light intensity transmission through a $10 \mu \mathrm{m}$ thick slab was made, seen in Fig. 4. The purpose of this figure

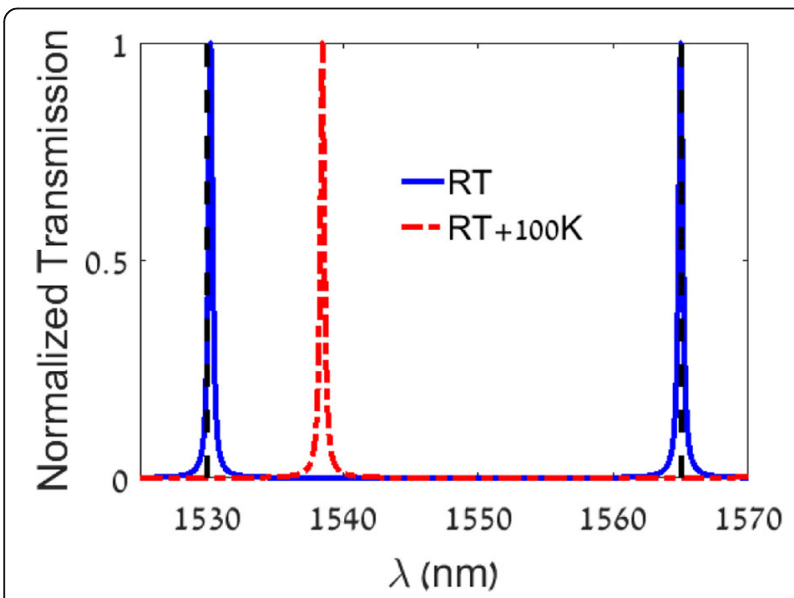

Fig. 4 Simulation for slab of $10 \mu \mathrm{m}$ which cover the entire C-band (done for finesse 100) is to show that a full C-band scan can be done with only one wavelength transmitted, i.e. channel separation can be accomplished for the full C-band, even though the transmitted spectrum is periodic, which could not be done for the entire C-band with a $470 \mu \mathrm{m}$ thick slab. This is achieved by the wide FSR of the slab, which is determined by its thickness.

In this figure, transmissions through coated Si slab vs. wavelength, in the range of $1520 \mathrm{~nm}$ to $1570 \mathrm{~nm}$, is seen at RT (solid blue) and at $100 \mathrm{~K}$ (dashed red). Since the maximum in transmission is periodic every about $35 \mathrm{~nm}$ (for a $10 \mu \mathrm{m}$ slab FSR), there are two maxima at RT (solid blue) in this graph range, at $1530 \mathrm{~nm}$ and at $1565 \mathrm{~nm}$. That is, at these two wavelengths of probe laser the transmission intensity through the slab will be at its maximum. However, when the slab is heated to $100 \mathrm{~K}$ the maximum in probe transmission will only be when the probe is lasing at $1540 \mathrm{~nm}$. With this transmission spectrum only one peak in transmission exist, rather than several peaks in thicker samples. Thus, only a specific wavelength can be chosen to be transmitted through the slab in the region of interest.

As mentioned above, different slab thicknesses are required for different regions of wavelengths (channels) scanning and spectral resolution. With the thinner slabs more channels are transmitted, in which the spectral resolution is higher, whereas with thicker slabs less channels are transmitted, but with lower spectral resolution. Thus, there is a tradeoff between thicker and thinner slabs. Whether to use one thickness or another is determined by the circumstances and objectives of the system. For instance, as shown above, for a full C-band scan with a single transmitted channel a slab of $10 \mu \mathrm{m}$ is required. However, the slabs at our disposal are of 470 and $50 \mu \mathrm{m}$ thick. Therefore, in the experimental results shown hereafter, several channels across the $\mathrm{C}$-band are transmitted with spectral resolution of up to $0.07 \mathrm{~nm}$. This resolution can be further increased with better finesse. 


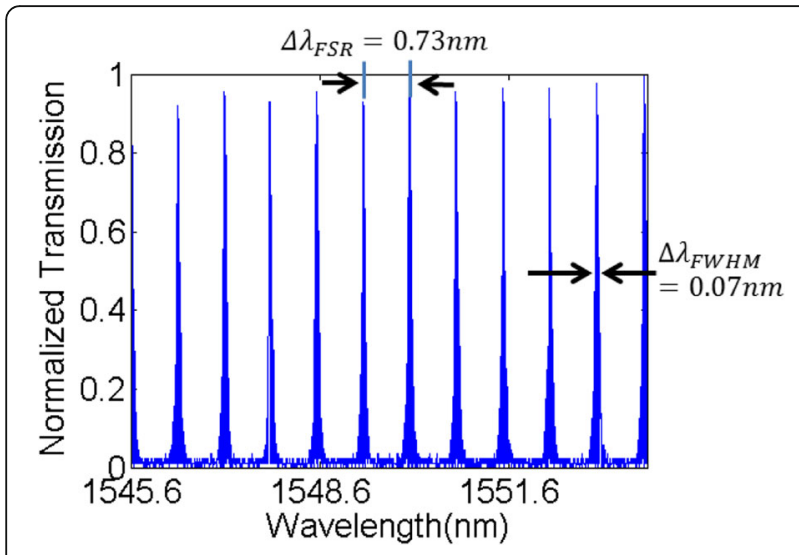

Fig. 5 Transmission of $470 \mu \mathrm{m}$ thick coated Si slab vs. wavelength

\section{Experimental results}

Transmission through the $\mathrm{Si}$ slab as a function of wavelength for about $8 \mathrm{~nm}$ in the C-band region can be seen in Fig. 5. The transmission spectrum is measured whilst the laser beam wavelength is scanned. The FSR of the $\mathrm{Si}$ slab is $0.73 \mathrm{~nm}$ and its FWHM is $0.07 \mathrm{~nm}$ which results from finesse of 10 . The transmission comb is represented as the "through transmission" rather than the "drop transmission" usually shown in ring resonators.

As mentioned above and shown in the simulations, the filter can be tuned by changing the temperature. Experimental results of the normalized transmission spectrum through the $\mathrm{Si}$ etalon as a function of wavelength at different temperatures can be seen in Fig. 6a. A shift in the transmission spectrum to longer wavelengths is induced, according to the TOE theorem, due to an increase in the temperature. The shift is of about $0.124 \mathrm{~nm}$ which relates to an increase in the real refractive index of $2.6 \mathrm{E}-4$. The latter is induced by an increase in the slab temperature of $1.4 \mathrm{~K}$. From these results, the linear relation between the wavelength shift and temperature is calculated to be $0.089 \mathrm{~nm} / \mathrm{K}$, which is not far from the simulation prediction of $0.083 \mathrm{~nm} / \mathrm{K}$.

For a slab of $470 \mu \mathrm{m} \Delta T_{\pi}$ is about $9 \mathrm{~K}$. Even though the temperature for a full FSR scan is not high and therefore can be attained fast and with low power consumption relative to thinner slabs, its FSR is not broad enough to cover the entire $\mathrm{C}$-band region. Notwithstanding, the filter FWHM is $0.07 \mathrm{~nm}$ or about $9 \mathrm{GHz}$ and can provide a good channel isolation. The attenuation factor or extinction ratio, as one of the features which define the quality of the filter, is about $20 \mathrm{~dB}$. In Fig. $6 \mathrm{~b}$ one can see the summary of several wavelength shifts with temperature in which a linear behavior is observed; the experimental results (red circles) fit well with the simulation (solid blue line). The temperature is in the range of $10 \mathrm{~K}$, a range that is useful in feedback loops and DWDM wavelength shifts, as mentioned in the method section.
As seen above, in Fig. 6, the wavelength shift as a function of temperature has a linear behavior. In order to discover how the wavelength shift as a function of wavelength behaves, an experiment was conducted, as seen in Fig. 7. In this figure, the intensity transmission was measured as a function of wavelength at $30{ }^{\circ} \mathrm{C}$ (blue solid line) and at $33^{\circ}$ $\mathrm{C}$ (red dashed line) over the wavelength range of about $10 \mathrm{~nm}$. The results show that the wavelength shift as a function of the wavelength in that range is close to constant, around $0.25 \mathrm{~nm} \pm 0.005 \mathrm{~nm}$ with $\Delta \mathrm{T}=3 \mathrm{~K} . \mathrm{nm}$ The $\pm 0.005 \mathrm{~nm}$ variation along $10 \mathrm{~nm}$ is an order of magnitude less than the aforementioned resolution of $0.07 \mathrm{~nm}$, that is, the accuracy is good.

The FSR, and therefore the FWHM as a nominal finesse, depends on the thickness of a slab, thus, a different slab thickness behavior with temperature is in order. In Fig. 8 transmission as a function of wavelength through $50 \mu \mathrm{m}$ coated silicon slab is seen. One can see that the $6.9 \mathrm{~nm}$ FSR is wider than in the $470 \mu \mathrm{m}$ thick slab, due to the increased thickness, as predicted in the simulation section above. In addition, the $0.5 \mathrm{~nm}$ FWHM of the etalon which is narrower than the $470 \mu \mathrm{m}$ slab indicates on a greater finesse and allegedly a better resolution in channel selection. Notwithstanding, the FWHM is still broader than the thicker slab, so even though the absolute resolution of the $470 \mu \mathrm{m}$ slab is better, the wider FSR of the $50 \mu \mathrm{m}$ slab yields an broader differentiation between channels.

As mentioned above, the simplicity of the proposed device gives it an advantage over other IIR filters such as ring resonators by enabling it to be integrated between two fibers, avoiding the SOI structures and coupling of tapered waveguides. In Fig. 9a, a picture simplifying the idea of such a device in its preliminary form of integration, is presented and its operational scheme is shown in Fig. 9b. As the temperature is changed by the TEC the transmission spectrum shifts similarly to the free space setup.

\section{Discussion}

As mentioned above, a full C-band scan of $35 \mathrm{~nm}$ requires a $10 \mu \mathrm{m}$ thick silicon slab. In this thickness the $\Delta \mathrm{T} \pi$ is around $350 \mathrm{~K}$ above $\mathrm{RT}$. This temperature requires consideration of the thermal parameters such as the expansion coefficient and the $\Delta \mathrm{T} \pi$. The former affects the FSR; however, in that temperature it is negligible, less than pico-meter change. The latter, although depending on the thermal expansion coefficient, is mainly determined by the TOE which also affects the FSR. However, the TOE is around 20 times larger than the thermal expansion in the temperature range of $350 \mathrm{~K}$. Therefore, the TOE dependence on temperature is taken into account in eq. (1), whereas the thermal expansion coefficient change can be neglected.

One can notice that the experimental results shown in Figs. 5, 6, 7 and 8 are noisy in addition to the changes in 


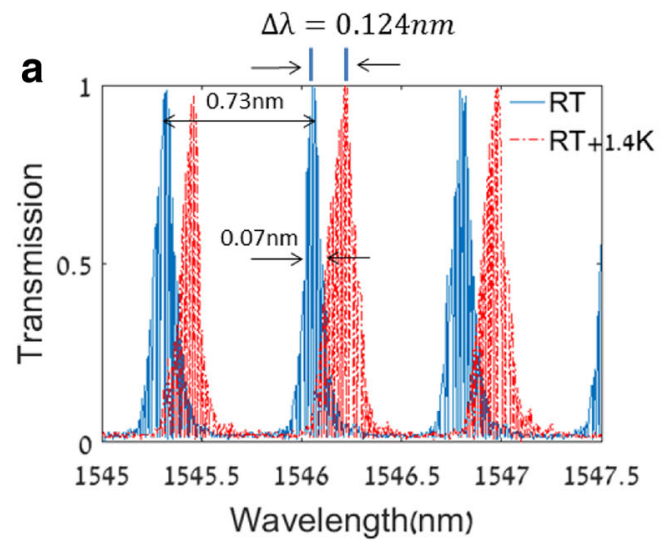

b

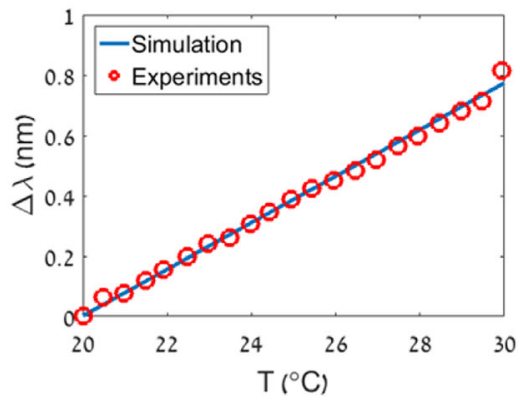

Fig. 6 a Transmission spectrum of $470 \mu \mathrm{m}$ thick coated Si vs. wavelength at RT (in solid blue line) and at RT $+1.4 \mathrm{~K}$ (in dashed red line). $\mathbf{b}$ The wavelength shifts vs. temperature range of $10 \mathrm{~K}$

FWHM, in Fig. 6a which is up to $50 \%$ in the FSR range. The measured transmission spectrum is constructed from of relatively large discrete wavelength scanning steps, of $10 \mathrm{pm}$, of the tunable probe laser. There is a tradeoff between small steps, which take more scanning time, but result in higher transmission spectrum resolution and large steps, which take less scanning time but produce lower transmission spectrum resolution. Furthermore, the changes in FWHM of up to 50\%, in the FSR range, seen in Fig. $6 \mathrm{a}$ are ascribed to noise. Any noise in the system may be due to temperature fluctuations. In addition, due to sample thickness variation, the transmission spectrum changes when the probe is transmitted through different locations in the slab, so any mechanical vibration or instability might cause changes in the transmission spectrum. Therefore, spatial uniformity is an important

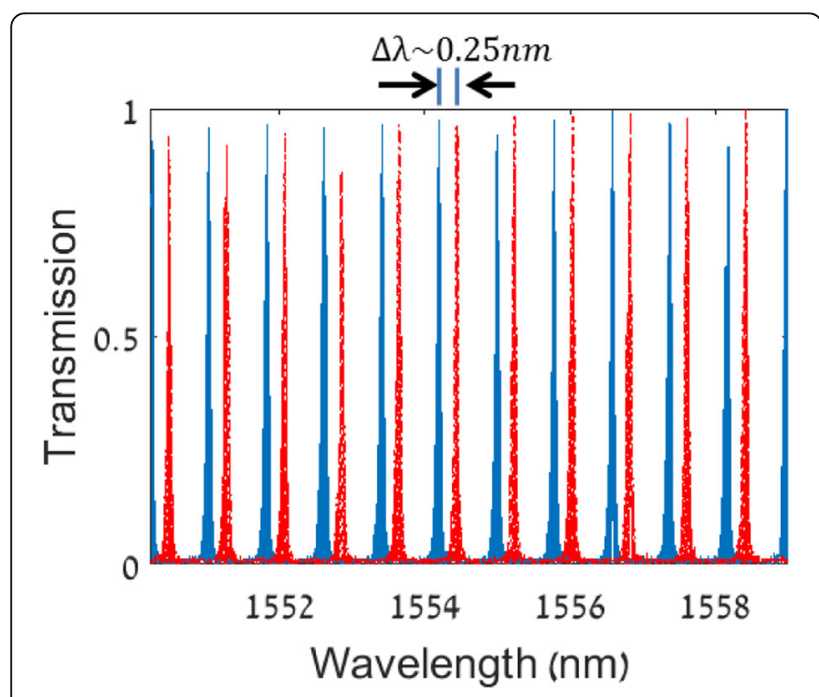

Fig. 7 Transmission spectrum of $470 \mu \mathrm{m}$ thick coated Si slab vs. wavelength at $30{ }^{\circ} \mathrm{C}$ (blue solid line) and at $33^{\circ} \mathrm{C}$ (red dashed line) ingredient in such a method. However, all of the above can be better controlled and improved.

The device presented in this paper can act as a narrowband optical filter offering tunability in the C-band region with narrow and smooth spectral transmission window which provides good channel isolation. The narrow transmission window is often measured as the bandwidth which in this paper is shown to be $0.07 \mathrm{~nm}$ or almost $9 \mathrm{GHz}$. Furthermore, the bandwidth can be decreased below $3 \mathrm{GHz}$ by improving the finesse of the device: As shown in previous work [29], a finesse of 30 can be achieved which can yield the aforementioned narrow bandwidth. However, there is a tradeoff between greater channels separation in a $50 \mu \mathrm{m}$ thick slab to better resolution in a $470 \mu \mathrm{m}$ thick slab.

As seen above, this device is very sensitive to a change of temperature. While this is useful for switching, it can cause stabilization problems. Therefore, a temperature controller with specific parameters, such as temperature range, resolution, accuracy and stabilization is required to be fitted for each application. For instance, a full Cband filter system with $0.1 \mathrm{~nm}$ resolution will have to have a temperature controller with a temperature range between RT and RT $+350 \mathrm{~K}$ (with a $10 \mu \mathrm{m}$ thick slab) and a temperature resolution of $1 \mathrm{~K}$.

A full C-band scan requires $350 \mathrm{~K}$ increase in temperature which results in several arising issues mentioned next. One of them is that at this high temperature of $380{ }^{\circ} \mathrm{C}$ the forbidden gap might change and so the absorption will increase, however, it remains around $\sim 1.1 \mathrm{eV}$ so the absorption will not change significantly. Another issue is the film and housing stability which will have to be suited for the application in terms of stability requirements in this high temperature. Future developments can be integrating the device inside a vacuum chamber with a $3 \mathrm{D}$ structure for temperature control. In the device shown in this paper the power consumption was not given much weight. However, taking it into 


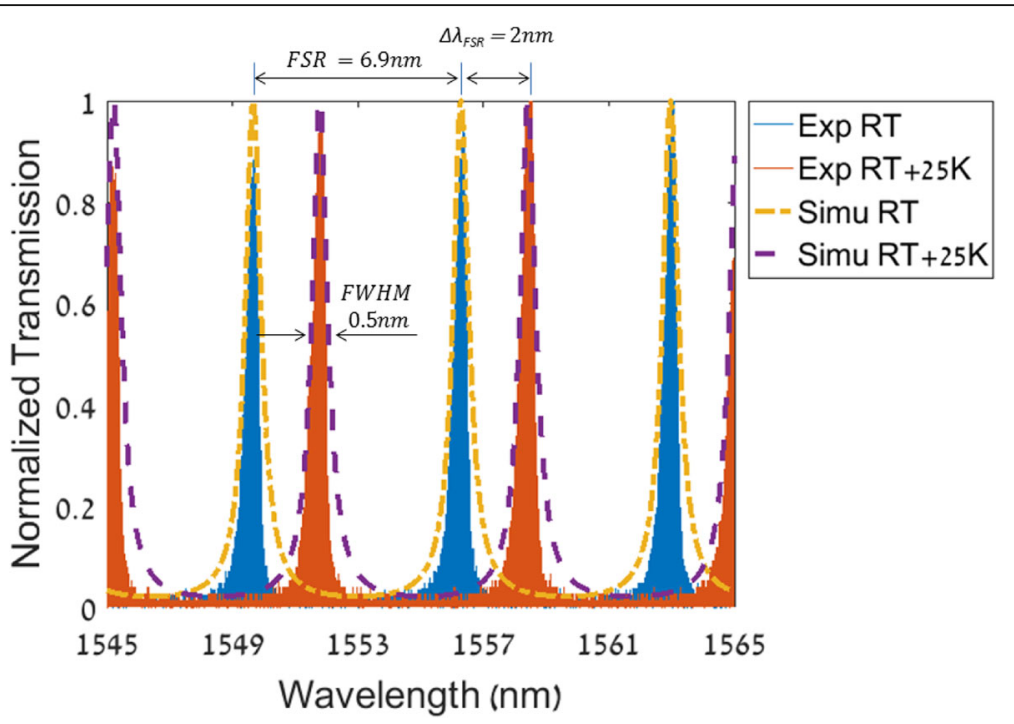

Fig. 8 Transmission of a $50 \mu m$ thick coated Si slab vs. wavelength at RT and at RT + $25 \mathrm{~K}$, experiment and simulation

account, the maximum power consumption is dependent on the silicon volume needed to be heated and can be as low as the order of $10 \mathrm{~mW}$. The last issue is the maximal temperature which can decrease if the initial temperature will be below room temperature and the device calibrated accordingly, for instance, between $0{ }^{\circ} \mathrm{C}$ and $350{ }^{\circ} \mathrm{C}$.

Since the transmission spectrum is periodic, there is a problem of choosing and knowing which tooth of the comb will transmit the target wavelength. A solution to this problem is as follows: By calibrating the system to match a specific channel for each temperature, any wavelength transmission in the C-band region can be accurately achieved. As seen in the full C-band scan simulation

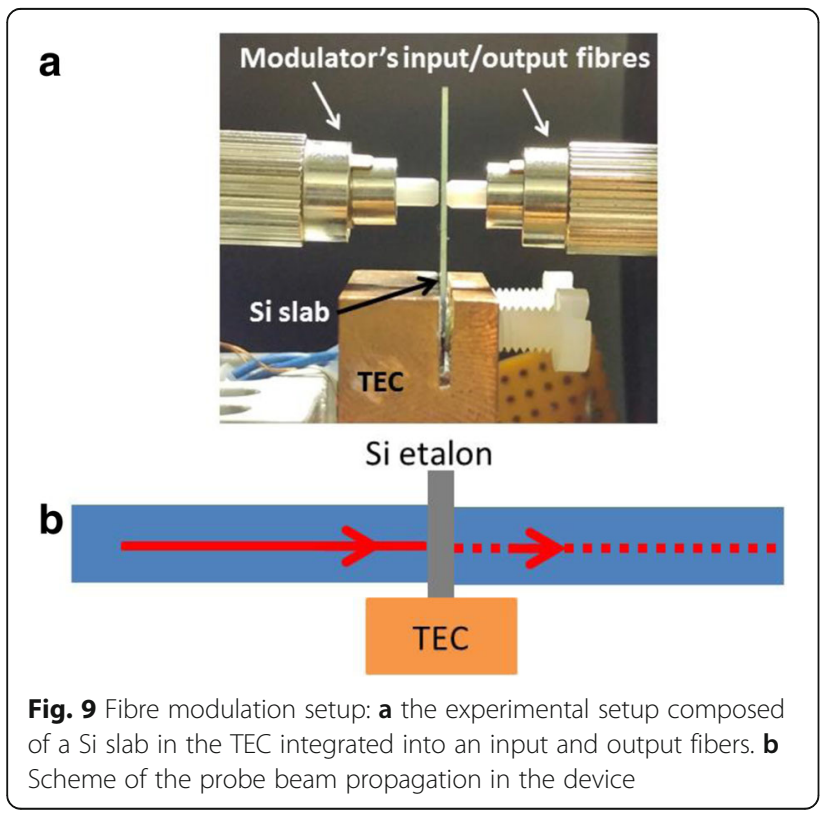

result, Fig. 4, a $10 \mu \mathrm{m}$ silicon slab is sufficient for a full C-band scan, that is, a $35 \mathrm{~nm}$ scan. Calibration can be done by one time recording of the transmitted wavelength at each temperature, for example, between RT and $\mathrm{RT}+350 \mathrm{~K}$. Once it is done for the entire C-band the wavelength can be recalled according to recorded wavelength at that temperature. Since the $10 \mu \mathrm{m}$ FSR is $35 \mathrm{~nm}$, a finesse of 100, resulting in FWHM of $0.35 \mathrm{~nm}$, will answer the need of $0.4 \mathrm{~nm}$ DWDM channel isolation. Therefore, our device is suitable for discrete channel switching in optics communication applications.

Implementation the filter between two fibers (Fig. 9) will induce more than $3 \mathrm{~dB}$ signal loss due to insertion loss. However, this configuration is only for simplification of the idea. For low loss applications the integration will have to be implemented in different ways. One way is to use collimators at the input and output of the fibers. Another way is shown in [8], in which they propose a compact all-fiber integrated modulator in which a free space cavity is replaced with a section of the fiber and constructed from a silicon core waveguide surrounded by a silica cladding and present a $1 \mathrm{~dB}$ insertion loss. They also discuss the device polarization independence as is the case in this paper. Therefore, there is no loss due to varying polarizations.

Optical applications often require repeatable, accurate and fast tunable filter such as closed feedback loop. For these applications a short bandwidth tunable filter that is implemented as an all-optical modulator or all-optical switching device can fulfil this need. This possibility of answering this need is shown in the work previously mentioned which presents this implementation by introducing some characteristics of our device such as speed (nanoseconds), accuracy and repeatability. 
In addition, these capabilities make this device suitable for optical communication systems such as DWDMs. These multiplexers are profuse with channels and therefore have short channel spacing. However, receivers, in contrast to laser sources, prone to be wideband devices, thus, presenting a problem in channel de-multiplexing. Therefore, tunable filter which can switch channels in the C-band with $9 \mathrm{GHz}$ and even up to $3 \mathrm{GHz}$ (in larger finesse) is a solution for ever-growing number of channels in the C-band.

Our device has a smooth, rounded transmission spectrum that is the result of a single FP resonator, or cavity. However, flat top bandpass filters can be made by stacking multiple cavities together. The stacking of cavities results in the increase of the roll-off slope which in turn improves the out-of-band rejection level. Thus, our device, implemented as a multi-cavities device, can be used as an efficient optical comb switching for multiwavelength message routing in Si photonics network applications such as CMOS technologies.

\section{Conclusions}

In this paper, we presented tunable thermo-optic filtering device based on coated silicon slab resonator with increased Q-factor for the C-band optical switching. It has a narrow and smooth transmission window which provides good channel isolation. Therefore, this device can be designed either for long range wavelength tuning of for short range with increased wavelength resolution. Proper channel isolation with an extinction ratio of $20 \mathrm{~dB}$ is achieved with spectral bandpass width of $0.07 \mathrm{~nm}$. In addition, the coatings enable it to be fabricated in in-fiber applications such as integrated in-fiber optics communication system filter in addition to its simplicity. The characteristics of the proposed device make it suitable for wavelength switching systems such as DWDM.

\section{Abbreviations \\ DWDM: dense wavelength division multiplexing; FCCs: free charge carriers; FIR: finite impulse response; FP: Fabry-Perot; FSR: free spectral range; FWHM: full width half maximum; IIR: Infinite Impulse Response; RT: room temperature; SOI: Silicon-On-Insulator; TEC: thermo-electric cooler; TOC: thermo-optic coefficient; TOE: thermo-optic effect}

\section{Acknowledgements}

We thank Mr. Dov Fridman of the mechanical workshop at Bar-llan University for his technical support, expertise and willingness that greatly assisted the research.

\section{Funding}

Not applicable.

\section{Availability of data and materials}

The data will not be shared because of future developments and industrial applications which naturally require exclusivity and classifications, with the exception of reviewers' special request.

\section{Authors' contributions}

HP has conducted experiments and reached conclusions. DM has come up with the idea. YD is the science consultant and producer of Collaborations. MS is the physical consultant and has provided guidance. $Z Z$ is the theoretical consultant and has provided guidance. All authors read and approved the final manuscript.

Ethics approval and consent to participate

Not applicable.

Consent for publication

Not applicable.

\section{Competing interests}

The authors declare that they have no competing interests.

\section{Publisher's Note}

Springer Nature remains neutral with regard to jurisdictional claims in published maps and institutional affiliations.

\section{Author details}

${ }^{1}$ Faculty of Engineering and the Nano-Technology Center, Bar-llan University, 5290002 Ramat Gan, Israel. ${ }^{2}$ Faculty of Engineering, Holon Institute of Technology (HIT), 5810201 Holon, Israel.

Received: 11 July 2017 Accepted: 24 October 2017

Published online: 02 November 2017

\section{References}

1. Yariv, A., Yeh, P.: Photonics, 6th Ed. Oxford University Press (2007)

2. Rabus, D.G., Hamacher, M., Troppenz, U., Heidrich, H.: Optical filters based on ring resonators with integrated semiconductor optical amplifiers in GalnAsP-InP. IEEE J. Sel. Top. QUANTUM Electron. 8(6), (2002)

3. Madsen, C.K., Zhao, J.H.: Optical Filter Design and Analysis : a Signal Processing Approach. John Wiley (1999)

4. Orta, R., Savi, P., Tascone, R., Trinchero, D.: Synthesis of multiple-ring resonator filters for optical systems. IEEE Photon. Technol. Lett. 7(12), 14471449 (Dec. 1995)

5. Griffel, G.: Synthesis of optical filters using ring resonator arrays. IEEE Photon. Technol. Lett. 12(7), 810-812 (Jul. 2000)

6. Bogaerts, W., et al.: Silicon microring resonators. Laser Photonics Rev. 6(1), 47-73 (2012)

7. Almeida, V.R., Barrios, C.A., Panepucci, R.R., Lipson, M.: All-optical control of light on a silicon chip. Nature. 431(7012), 1081-1084 (Oct. 2004)

8. R. Aharoni, O. Baharav, L. Bidani, M. Sinvani, D. Elbaz, and Z. Zalevsky, "Alloptical silicon simplified passive modulation," J. Eur. Opt. Soc. - Rapid Publ., vol. 7, no. 12029, Jul. 2012

9. H. Pinhas, L. Bidani, O. Baharav, M. Sinvani, M. Danino, and Z. Zalevsky, "All optical modulator based on silicon resonator," in SPIE, 2015, vol. 9609, p. 96090L-96090L-7

10. G. Cocorullo, F. G. Della Corte, M. lodice, and I. Rendina, "Simple and lowcost silicon Fabry-Perot filter for WDM channel monitoring," in 2000 Digest of the LEOS Summer Topical Meetings. Electronic-Enhanced Optics. Optical Sensing in Semiconductor Manufacturing. Electro-Optics in Space. Broadband Optical Networks (Cat. No.00TH8497), 2000, pp. IV45-IV46

11. Iodice, M., Cocorullo, G., Della Corte, F., Rendina, I.: silicon Fabry-Perot filter for WDM systems channels monitoring. Opt. Commun. 183(5-6), 415-418 (Sep. 2000)

12. Liu, Z., Liu, Z., Deng, Z., Tao, L.: Interference signal frequency tracking for extracting phase in frequency scanning interferometry using an extended Kalman filter. Appl. Opt. 55(11), 2985 (Apr. 2016)

13. N. European Exhibition on Optical Communication 2nd: Oslo, 22nd European Conference on Optical Communication, ECOC 96 : September 1519, 1996, Oslo, Norway, "Folkets Hus" Congress Centre. Telenor R \& D, 1996

14. Ben Zaken, B., Zanzury, T., Malka, D.: An 8-channel wavelength MMl Demultiplexer in slot waveguide structures. Materials (Basel). 9(11), 881 (Nov. 2016)

15. Malka, D., Peled, A.: Power splitting of $1 \times 16$ in multicore photonic crystal fibers. Appl. Surf. Sci. 417, 34-39 (2017)

16. Malka, D., Cohen, M., Turkiewicz, J., Zalevsky, Z.: Optical micro-multi-racetrack resonator filter based on SOI waveguides. Photonics Nanostructures Fundam. Appl. 16, 16-23 (2015)

17. Andrews, N.L.P., et al.: In-fiber Mach-Zehnder interferometer for gas refractive index measurements based on a hollow-core photonic crystal fiber. Opt. Express. 24(13), 14086 (Jun. 2016) 
18. Treyz, G.V.: Silicon Mach-Zehnder waveguide interferometers operating at 1. $3 \mu \mathrm{m}$. Electron. Lett. 27(2), 118 (1991)

19. Cortelazzo, G., Lightner, M.: Simultaneous design in both magnitude and group-delay of IIR and FIR filters based on multiple criterion optimization. IEEE Trans. Acoust. 32(5), 949-967 (Oct. 1984)

20. Palupi, R.R., Syahriar, A., Lubis, A.H., Rahardjo, S.: Sardjono, simulation of Mach Zehnder Interleaver based thermo-optic effect in L-band range. In: RSM 2013 IEEE Regional Symposium on Micro and Nanoelectronics, pp. 269-272 (2013)

21. Okada, Y., Tokumaru, Y.: Precise determination of lattice parameter and thermal expansion coefficient of silicon between 300 and $1500 \mathrm{~K}$. J. Appl. Phys. 56(2), 314-320 (Jul. 1984)

22. Lee, B.G., Biberman, A., Dong, P., Lipson, M., Bergman, K.: All-optical comb switch for multiwavelength message routing in silicon photonic networks. IEEE Photon. Technol. Lett. 20(10), 767-769 (May 2008)

23. Sun, X., et al.: Tunable silicon Fabry-Perot comb filters formed by Sagnac loop mirrors. Opt. Lett. 38(4), 567 (Feb. 2013)

24. Tobing, L.Y.M., Lim, D.C.S., Dumon, P., Baets, R., Chin, M.-K.: Finesse enhancement in silicon-on-insulator two-ring resonator system. In: Appl. Phys. Lett., vol. 92, no. 10, p. 101122 (Mar. 2008)

25. T. Kessler et al., "A Sub-40-mHz-Linewidth Laser Based on a Silicon SingleCrystal Optical Cavity," 2012

26. Gosciniak, J., Bozhevolnyi, S.I.: Performance of thermo-optic components based on dielectric-loaded surface plasmon polariton waveguides. Sci Rep. 3, 1246-1260 (May 2013)

27. Harris, N.C., et al.: Efficient, compact and low loss thermo-optic phase shifter in silicon. Opt. Express. 22(9), 10487 (May 2014)

28. Prabhathan, P., Jing, Z., Murukeshan, V.M., Huijuan, Z., Shiyi, C.: Discrete and fine wavelength tunable Thermo-optic WSS for low power consumption C +L band Tunability. IEEE Photon. Technol. Lett. 24(2), 152-154 (Jan. 2012)

29. Pinhas, H., Danan, Y., Sinvani, M., Danino, M., Zalevsky, Z.: Experimental characterization towards an in-fibre integrated silicon slab based all-optical modulator. J. Eur. Opt. Soc. Publ. 13(1), 3 (Dec. 2017)

30. Soref, R.A., Bennett, B.R.: Electrooptical effects in silicon. IEEE J. Quantum Electron. 23(1), 123-129 (Jan. 1987)

31. Cocorullo, G., Rendina, I.: Thermo-optical modulation at $1.5 \mu \mathrm{m}$ in silicon etalon. Electron. Lett. 28(1), 83-85 (Jan. 1992)

32. Della Corte, F.G., Montefusco, M.E., Moretti, L., Rendina, I., Cocorullo, G.: Temperature dependence analysis of the thermo-optic effect in silicon by single and double oscillator models. J. Appl. Phys. 88(12), 7115 (2000)

33. Grillanda, S., et al.: Non-invasive monitoring and control in silicon photonics using CMOS integrated electronics. Optica. 1(3), 129 (Sep. 2014)

34. Tinker, M.T., Lee, J.-B.: Thermal and optical simulation of a photonic crystal light modulator based on the thermo-optic shift of the cut-off frequency. Opt. Express. 13(18), 7174 (Sep. 2005)

35. C. A. Barrios, V. R. de Almeida, and M. Lipson, "Low-power-consumption short-length and high-modulation-depth silicon electrooptic modulator," J. Light. Technol., vol. 21, no. 4, pp. 1089-1098, Apr. 2003

\section{Submit your manuscript to a SpringerOpen ${ }^{\circ}$ journal and benefit from:}

- Convenient online submission

- Rigorous peer review

- Open access: articles freely available online

- High visibility within the field

- Retaining the copyright to your article 